\title{
THE VANISHING OF POINCARÉ SERIES
}

\author{
by R. A. RANKIN \\ (Received 4th September 1978)
}

\section{Introduction}

Every holomorphic modular form of weight $k>2$ is a sum of Poincaré series; see, for example, Chapter 5 of (5). In particular, every cusp form of even weight $k \geqq 4$ for the full modular group $\Gamma(1)$ is a linear combination over the complex field $\boldsymbol{C}$ of the Poincaré series

$$
G_{k}(z, m)=\frac{1}{2} \sum_{T}(c z+d)^{-k} \exp \{2 \pi i m T(z)\}
$$

Here $\boldsymbol{m}$ is any positive integer, $z \in \boldsymbol{H}=\{z \in \boldsymbol{C}: \operatorname{Im} z>0\}$ and

$$
T(z)=\frac{a z+b}{c z+d}
$$

The summation is over all matrices

$$
T=\left[\begin{array}{ll}
a & b \\
c & d
\end{array}\right]
$$

with different second rows in the (homogeneous) modular group, i.e. in $S L(2, Z)$. The factor $\frac{1}{2}$ is introducted for convenience.

When $k=4,6,8,10$ and 14 , the space $C_{k}$ of cusp forms has dimension zero, so that, in each of these five cases, $G_{k}(z, m)$ vanishes identically for all positive integers $m$. We write

$$
\mu_{k}=\operatorname{dim} C_{k} \text {. }
$$

Then $\mu_{k}>0$ for $k=12$ and all even $k \geqq 16$. In fact (5, Theorem 6.1.2), for $k \geqq 4$,

$$
\mu_{k}=\left\{\begin{array}{lll}
{\left[\frac{k}{12}\right]} & \text { if } & k \neq 2(\bmod 12), \\
{\left[\frac{k}{12}\right]-1} & \text { if } & k \equiv 2(\bmod 12)
\end{array}\right\}
$$

It follows that $\mu_{k}=O(k)$ for large $k$.

Moreover, when $\mu_{k} \geqq 1$, the series $G_{k}(z, m)\left(1 \leqq m \leqq \mu_{k}\right)$ span the space $C_{k}$, so that, in particular,

$$
G_{k}(z, m) \not \equiv 0 \text { for } 1 \leqq m \leqq \mu_{k}
$$

See, for example, Theorem 6.2.1 of (5).

The object of the present paper is to consider whether (1.4) can be extended to values of $m$ greater than $\mu_{k}$, when $k$ is large. 
It may be noted that, since, as shown in $\$ 6.3$ of (5),

$$
G_{12}(z, m)=c_{12} m^{11} \tau(m) \Delta(z),
$$

where $\Delta$ is the discriminant function, $\tau(m)$ is Ramanujan's coefficient, and $c_{12}$ is a positive constant, more information is available in this particular case. For Lehmer has shown that $\tau(m) \neq 0$ for

$$
1 \leq m \leqq 113740230287998
$$

and, accordingly, $G_{12}(z, m) \not \equiv 0$ for these values of $m$; see $\$ 6.6$ of (5). Formulae similar to (1.5) hold also for $G_{k}(z, m)$ when $k=16,18,20,22$ and 26.

We prove the following theorem.

Theorem 1. There exist positive constants $k_{0}$ and $B$, where $B>4 \log 2$, such that, for all even $k \geqq k_{0}$ and all positive integers

$$
m \leqq k^{2} \exp \{-B \log k / \log \log k\},
$$

the Poincaré series $G_{k}(z, m)$ does not vanish identically.

Note that the theorem remains true for arbitrary $\varepsilon>0$ and suitably large $k_{0}$ if the right-hand side of (1.6) is replaced by $k^{2-\varepsilon}$.

The proof uses sharp estimates for the magnitude of Kloosterman sums. If cruder approximations are used, it is easy to replace $k^{2-\varepsilon}$ by the weaker $k^{3 / 2}$. The method can also be applied to Poincaré series belonging to congruence subgroups of the modular group.

Some additional results concerning the vanishing or non-vanishing of particular subsets of Poincaré series are obtained in $\$ 6$.

Throughout the paper $A_{1}, A_{2}, \ldots, B_{1}, B_{2}, \ldots$ denote positive absolute constants; in particular, these parameters do not depend upon the weight $k$.

\section{Preliminaries}

It is convenient to follow Petersson (3) and introduce the function

$$
g_{k}(z, m)=m^{k-1} G_{k}(z, m) .
$$

We shall always assume that $m$ and $k$ are positive integers, the latter being even. Write

$$
g_{k}(z, m)=\sum_{r=1}^{\infty} c_{k}(r, m) e^{2 \pi i r z}
$$

where $z \in \boldsymbol{H}$. Then it is known that

$$
c_{k}(r, m)=(r m)^{(k-1) / 2}\left\{\delta_{r, m}+2 \pi(-1)^{k / 2} \sum_{q=1}^{\infty} \frac{S(r, m ; q)}{q} J_{k-1}\left(\frac{4 \pi \sqrt{ }(r m)}{q}\right)\right\},
$$

where $\delta_{r, m}$ is the Kronecker delta, $J_{k-1}$ is the Bessel function of the first kind, and $S(r, m ; q)$ is the Kloosterman sum

$$
S(r, m ; q)=\sum_{\substack{h=1 \\(h, q)=1}}^{q} \exp \left\{\frac{2 \pi i}{q}\left(r h+m h^{\prime}\right)\right\}
$$

here $h h^{\prime} \equiv 1(\bmod q)$. See $(4)$ or Theorem 5.3 .2 of $(5) ;(2.3)$ is a particular case of a result first proved by Petersson in 1931. 
Since $S(r, m ; q)=S(m, r ; q)$, it is clear that

$$
c_{k}(r, m)=c_{k}(m, r)
$$

Moreover, since, by (6.2.7) in (5),

$$
\left(g_{k}(z, r), g_{k}(z, m)\right)=\omega_{k} c_{k}(r, m),
$$

where the left-hand side denotes the Petersson inner pruduct and $\omega_{k}$ is positive, it follows that $c_{k}(r, m)$ is real and that

$$
c_{k}(m, m) \geqq 0 \text {. }
$$

Further, we deduce, as Petersson did (3), that $g_{k}(z, m)$, and there ${ }^{\mathfrak{f}}$ ore $G_{k}(z, m)$ vanishes identically if and only if $c_{k}(m, m)=0$. For this reason we use as our starting point the formula

$$
c_{k}(m, m)=m^{k-1}\left\{1+2 \pi(-1)^{k / 2} \sum_{q=1}^{\infty} \frac{S(m, m ; q)}{q} J_{k-1}\left(\frac{4 \pi m}{q}\right)\right\}
$$

\section{Estimates of certain arithmetical functions}

Kloosterman sums have certain additive properties from which corresponding properties of the coefficients $c_{k}(r, m)$ can be deduced. For example, from the formula

$$
S\left(r p^{\rho}, m p^{\mu} ; q\right)=S\left(r, m p^{\rho+\mu} ; q\right)+p S\left(r p^{\rho-1}, m p^{\mu-1} ; q / p\right)
$$

which holds for any prime $p$ and positive integers $q, r, m, \rho, \mu$ satisfying

$$
p \mid q, \quad p+r, \quad p+m
$$

we easily deduce that, under the same conditions,

$$
c_{k}\left(r p^{\rho}, m p^{\mu}\right)=c_{k}\left(r, m p^{\rho+\mu}\right)+p^{k-1} c_{k}\left(r p^{\rho-1}, m p^{\mu-1}\right) .
$$

We use this result in $\S 6$.

Further, if $\left(q_{1}, q_{2}\right)=1$, then

$$
S\left(u, v ; q_{1} q_{2}\right)=S\left(u, v \bar{q}_{2}^{2} ; q_{1}\right) S\left(u, v \bar{q}_{1}^{2} ; q_{2}\right)
$$

where $q_{1} \bar{q}_{1} \equiv 1\left(\bmod q_{2}\right)$ and $q_{2} \bar{q}_{2} \equiv 1\left(\bmod q_{1}\right)$. The estimation of $S(r, m ; q)$ therefore reduces to the estimation of $S\left(r, m ; p^{n}\right)$, where $p$ is a prime and $n$ is a positive integer.

From Lemma 8 of Estermann's paper (1), and from the proof of his Lemma 9, we deduce that

$$
\left|S\left(r, m ; p^{n}\right)\right| \leqq 2^{\alpha} p^{(n+h) / 2} \quad(p \text { odd }),
$$

where $p^{h}=\left(r, m, p^{n}\right)$ and

$$
\alpha=0(h=n), \quad \alpha=1 \quad(h<n) .
$$

When $p=2$, we have, similarly,

$$
\left|S\left(r, m ; 2^{n}\right)\right| \leqq 2^{3 \alpha / 2} \cdot 2^{(n+h) / 2},
$$

where $2^{h}=\left(r, m, 2^{n}\right)$. This result improves slightly Lemma 3 of $(\mathbf{1})$ (where $\frac{3}{2} \alpha$ is replaced by $\left.\frac{5}{2} \alpha\right)$ and is easily deduced by a more efficient application of Lemma 2 of that paper. 
From $(3.3,4,5)$ we easily deduce

Lemma 3.1. Let $d=(q, r, m)$. Then

where

$$
|S(r, m ; q)| \leqq 2^{\omega(q / d)} q^{1 / 2} d^{1 / 2}
$$

$$
\omega(n)=\sum_{\left.p\right|_{n}} \omega(p) \quad \text { (p prime) }
$$

and where $\omega(2)=\frac{3}{2}$ and $\omega(p)=1(p>2)$.

It is easy to deduce from the Prime Number Theorem that there exists a positive constant $B_{1}>\log 2$ such that

where $x \geqq 2$.

$$
2^{\omega(n)} \leqq \exp \left(\frac{B_{1} \log x}{\log \log 2 x}\right)=: M(x) \text { for } n \leqq x,
$$

Now let, for any real $\lambda$,

$$
\sigma_{\lambda}(n)=\sum_{\substack{d \mid n \\ d>0}} d^{\lambda}
$$

We shall require upper bounds for $\sigma_{0}(n)$ and $\sigma_{-\frac{1}{2}}(n)$. It is easily deduced from the Prime Number Theorem that

$$
\sigma_{0}(m) \leqq M(m) \text { for } m \geqq 2 \text {, }
$$

and that, for some $B_{2}>2$,

$$
\sigma_{-1 / 2}(m) \leqq \exp \left\{\frac{B_{2}(\log m)^{1 / 2}}{\log \log 2 m}\right\} \quad(m \geqq 2) .
$$

\section{Bessel functions}

Write

$$
\nu=k-1 \text {. }
$$

We shall assume that $k \geqq 16$ so that $\nu \geqq 15$; as fractional powers of $\nu$ will occur it is convenient to write

$$
\sigma=\nu^{-1 / 6}
$$

We obtain upper bounds for $J_{\nu}(\nu x)$ valid for all $\nu \geqq 15$ and all $x \geqq 0$. These,involve various positive constants (independent of $\nu$ and $x$ ), which we denote by $A_{1}, A_{2}, \ldots$.

Lemma 4.1. For all $x \geqq 0$ and $\nu \geqq 15$,

$$
\left|J_{\nu}(\nu x)\right| \leqq(2 \pi \nu)^{-1 / 2}\left(\frac{1}{2} e x\right)^{\nu}
$$

Proof. The upper bound is, in fact, valid for $\nu \geqq 1$. We start from Poisson's integral

$$
J_{\nu}(\nu x)=\frac{2\left(\frac{1}{2} \nu x\right)^{\nu}}{\Gamma\left(\frac{1}{2}\right) \Gamma\left(\nu+\frac{1}{2}\right)} \int_{0}^{\pi / 2} \cos (\nu x \cos \theta) \sin ^{2 \nu} \theta d \theta
$$


see formula (5) on p. 48 of (7). Hence

$$
\left|J_{\nu}(\nu x)\right| \leqq \frac{2\left(\frac{1}{2} \nu x\right)^{\nu}}{\Gamma\left(\frac{1}{2}\right) \Gamma\left(\nu+\frac{1}{2}\right)} \int_{0}^{\pi / 2} \sin ^{2 \nu} \theta d \theta=\frac{\left(\frac{1}{2} \nu x\right)^{\nu}}{\nu !}
$$

from which the required result follows, since, for $\nu \geqq 1$,

Now put

$$
\nu ! \geqq \sqrt{ }(2 \pi) \nu^{\nu+1 / 2} e^{-\nu}
$$

and write

$$
x_{\nu}=\left(1-\sigma^{4}\right)^{1 / 2}, \quad y_{\nu}=\left(1+\sigma^{4}\right)^{1 / 2},
$$

$$
x=\operatorname{sech} \alpha \quad(0<x \leqq 1), \quad x=\sec \beta \quad(x \geqq 1) .
$$

Here $\alpha>0$ and $0 \leqq \beta \leqq \frac{1}{2} \pi$. We also write

$$
z=\nu(\alpha-\tanh \alpha) \quad(0<x \leqq 1), \quad z=\nu(\tan \beta-\beta) \quad(x \geqq 1) .
$$

Then, since $\nu \geqq 15$, it follows easily that

and

$$
z \geqq \frac{1}{3} \nu \tanh ^{3} \alpha \geqq \frac{1}{3} \quad\left(0<x \leqq x_{\nu}\right), \quad 0 \leqq z<\frac{2}{5} \quad\left(x_{\nu} \leqq x \leqq 1\right)
$$

$$
0 \leqq z \leqq \frac{1}{3} \quad\left(1 \leqq x \leqq y_{\nu}\right), \quad z>\frac{3}{10} \quad\left(y_{\nu} \leqq x\right)
$$

The parameters $\alpha$ and $\beta$ were introduced by Langer (2), but we have written $\nu$ for his $\rho$ and replaced $|\xi|$ by $z$.

Lemma 4.2. For $0<x \leqq 1$ and $\nu \geqq 15$,

$$
\left|J_{\nu}(\nu x)\right| \leqq \begin{cases}A_{1} \sigma^{3}\left(1-x^{2}\right)^{-1 / 4} \exp \left\{-\frac{1}{3} \nu\left(1-x^{2}\right)^{3 / 2}\right\} & \left(0<x \leqq x_{\nu}\right), \\ A_{1} \sigma^{2} & \left(x_{\nu} \leqq x \leqq 1\right) .\end{cases}
$$

Proof. For $x \leqq x_{\nu}$ we use formula (64) on p. 59 of (2). This express $J_{\nu}(\nu x)$ as an asymptotic series

$$
J_{\nu}(\nu \operatorname{sech} \alpha)=\frac{e^{-z}}{(2 \pi \nu \tanh \alpha)^{1 / 2}}\left\{1+O\left(\frac{1}{z}\right)\right\}
$$

for large $z$; note that here "large" means "bounded away from zero". From this and (4.6) we deduce (4.8).

For $x_{\nu} \leqq x \leqq 1, z$ is "small" by (4.6). We use formula (68) on p. 61 of (2), which shows that

$$
J_{\nu}(\nu \operatorname{sech} \alpha)=\frac{1}{\pi}\left(\frac{\alpha-\tanh \alpha}{\tanh \alpha}\right)^{1 / 2} K_{1 / 3}(z)+O\left(\nu^{-4 / 3}\right) .
$$

By Basset's formula on p. 172 of (7) for the modified Bessel function of the third kind,

so that

$$
K_{1 / 3}(z)=\frac{2^{1 / 3} \Gamma\left(\frac{5}{6}\right)}{z^{1 / 3} \sqrt{\pi}} \int_{0}^{\infty} \frac{\cos z u d u}{\left(u^{2}+1\right)^{5 / 6}}
$$

$$
\left|K_{1 / 3}(z)\right| \leqq \frac{2^{1 / 3} \Gamma\left(\frac{5}{6}\right)}{z^{1 / 3} \sqrt{\pi}} \int_{0}^{\infty} \frac{d u}{\left(u^{2}+1\right)^{5 / 6}}=\frac{\Gamma\left(\frac{1}{3}\right)}{(4 z)^{1 / 3}}
$$

From this (4.9) follows, since $\tanh \alpha \leqq \sigma^{2}$. 
Lemma 4.3. For $x \geqq 1$ and $\nu \geqq 15$

$$
\left|J_{\nu}(\nu x)\right| \leqq\left\{\begin{array}{l}
A_{2} \sigma^{2} \quad\left(1 \leqq x \leqq y_{\nu}\right) \\
A_{2} \sigma^{3}\left(x^{2}-1\right)^{-1 / 4} \quad\left(y_{\nu} \leqq x\right)
\end{array}\right.
$$

Proof. For the range $1 \leqq x \leqq y_{\nu}$ we note from (4.7) that $z$ is small and use formula (66)(a) on p. 60 of (2). This gives

$$
J_{\nu}(\nu \sec \beta)=\left(\frac{\tan \beta-\beta}{3 \tan \beta}\right)^{1 / 2}\left\{J_{1 / 3}(z)+J_{-1 / 3}(z)\right\}+O\left(\nu^{-4 / 3}\right) .
$$

Since $J_{\mu}(z)=O\left(z^{\mu}\right)$ for small $z,(4.10)$ follows.

For $x \geqq y_{\nu}$ we use formula (63) on p. 58 of (2), which shows that

$$
J_{\nu}(\nu \sec \beta)=\frac{2}{(\pi \nu \tan \beta)^{1 / 2}}\left\{\cos \left(z-\frac{\pi}{4}\right)+\phi\left(\frac{1}{z}\right)\right\},
$$

since, by (4.7), $z$ is large. From this (4.11) follows.

We now write $x_{0}$ for the positive root of the equation

$$
x \exp \left\{\frac{1}{3}\left(1-x^{2}\right)^{3 / 2}\right\}=2 e^{-1},
$$

so that $x_{0}=0.629$ approximately. We use Lemma 4.1 in the range $0 \leqq x \leqq x_{0}$ and Lemma 4.2 for $x_{0} \leqq x \leqq 1$.

It is convenient to define functions $f, F, g$ and $G$ as described below. We define $f$ on $[0,1]$ as follows:

$$
f(x)= \begin{cases}A_{3} \sigma^{3}\left(\frac{1}{2} e x\right)^{\nu} & \left(0 \leqq x \leqq x_{0}\right) \\ A_{4} \sigma^{3}\left(1-x^{2}\right)^{-1 / 4} \exp \left\{-\frac{1}{3} \nu\left(1-x^{2}\right)^{3 / 2}\right\} & \left(x_{0} \leqq x \leqq x_{\nu}\right) \\ \sigma^{2} & \left(x_{\nu} \leqq x \leqq 1\right)\end{cases}
$$

The positive constants $A_{3}$ and $A_{4}$ are chosen to make $f$ continuous on $[0,1]$; note that they are independent of $\nu$. Moreover $f(x)$, and therefore $x^{1 / 2} f(x)$, increase for $0 \leqq x \leqq 1$ and

$$
f(x) \leqq F(x) \quad(0 \leqq x<1)
$$

where $F(x)=f(x)$ for $0 \leqq x \leqq x_{\nu}$ and $F(x)$ is defined for $x_{\nu} \leqq x \leqq 1$ by the second formula in (4.13).

We define $g$ on $[0, \infty[$ by

$$
g(x)=\left\{\begin{array}{ll}
\sigma^{2} x^{1 / 2} & \left(1 \leqq x \leqq y_{\nu}\right) \\
\frac{\sigma^{3} x^{1 / 2}}{\left(x^{2}-1\right)^{1 / 4}} & \left(y_{\nu} \leqq x\right)
\end{array}\right\}
$$

Then $g$ is continuous and $g(x)$ increases for $1 \leqq x \leqq y_{\nu}$ and decreases for $y_{\nu} \leqq x$. Moreover

where

$$
g(x) \leqq G(x) \quad(x>1)
$$

$$
G(x)=\frac{\sigma^{3} x^{1 / 2}}{\left(x^{2}-1\right)^{1 / 4}} \quad(x>1) .
$$


From Lemmas 4.1, 4.2 and 4.3 we deduce

Lemma 4.4. A positive constant $A_{5}$ exists such that

and

$$
\left|J_{\nu}(\nu x)\right| \leqq A_{5} f(x) \text { for } 0 \leqq x \leqq 1
$$

$$
\left|x^{1 / 2} J_{\nu}(\nu x)\right| \leqq A_{5} g(x) \text { for } x \geqq 1 .
$$

\section{Proof of Theorem 1}

We consider the sum

$$
S_{m}=\sum_{q=1}^{\infty} \frac{S(m, m ; q)}{q} J_{k-1}\left(\frac{4 \pi m}{q}\right) .
$$

To estimate $S_{m}$ we use Lemma 3.1 for values of

$$
q<\frac{4 \pi m}{\nu}=Q
$$

and the estimate

$$
|S(m, m ; q)| \leqq q
$$

when $q \geqq Q$. We also put

$$
d=(q, m), \quad q=r d
$$

Then

where

$$
\left|S_{m}\right| \leqq S_{m}^{\prime}+S_{m}^{\prime \prime}
$$

$$
S_{m}^{\prime}=\sum_{d \mid m,} \sum_{r<Q / d} 2^{\omega(r)} r^{-1 / 2}\left|J_{\nu}\left(\frac{4 \pi m}{r d}\right)\right|
$$

and

$$
S_{m}^{\prime \prime}=\sum_{q \geqq Q}\left|J_{\nu}\left(\frac{4 \pi m}{q}\right)\right| .
$$

In view of what is known about $G_{12}(z, m)$, and since $G_{14}(z, m)$ vanishes identically for $m>0$, we may assume that $k \geqq 16$, so that $\nu \geqq 15$. We may also assume that

$$
m>\mu_{k} \text {. }
$$

It is then easily checked that

$$
m>\frac{4 \pi m}{15} \geqq Q \geqq \frac{4 \pi\left(\mu_{k}+1\right)}{k-1} \geqq \frac{8 \pi}{25}>1 .
$$

Accordingly, by (3.7) and (5.6),

$$
S_{m}^{\prime} \leqq \frac{M(m)}{Q^{1 / 2}} \sum_{\substack{d \mid m \\ d<O}} d^{1 / 2} T_{d}
$$


where, for $d \mid m$ and $d<Q$,

by (4.18).

$$
T_{d}=\sum_{1 \leqq r<Q / d}\left(\frac{Q}{r d}\right)^{1 / 2}\left|J_{\nu}\left(\frac{\nu Q}{r d}\right)\right| \leqq A_{5} \sum_{1 \leqq r<Q^{\prime} d} g\left(\frac{Q}{r d}\right),
$$

But, by the properties of the function $g$,

$$
\begin{aligned}
\sum_{1 \leqq r \leqq Q^{\prime} d} g\left(\frac{Q}{r d}\right) & \leqq \int_{1}^{Q / d} g\left(\frac{Q}{u d}\right) d u+2 g\left(y_{\nu}\right) \\
& =\frac{Q}{d} \int_{1}^{Q / d} x^{-2} g(x) d x+2 g\left(y_{\nu}\right) \\
& \leqq \frac{Q}{d} \int_{1}^{\infty} x^{-2} G(x) d x+2 \sigma^{2} y_{\nu}^{1 / 2} \\
& \leqq \frac{Q \sigma^{3}}{d} \int_{1}^{\infty} \frac{x^{-3 / 2} d x}{\left(x^{2}-1\right)^{1 / 4}}+3 \sigma^{2} \\
& =\frac{2(2 \pi)^{5 / 2}}{\Gamma^{2}\left(\frac{1}{4}\right)} \frac{m \sigma^{9}}{d}+3 \sigma^{2}
\end{aligned}
$$

Hence, by (5.9) and (5.10),

$$
\begin{aligned}
S_{m}^{\prime} & \leqq A_{6} M(m) \sum_{\substack{d \mid m \\
d<Q}}\left\{\left(\frac{m}{d}\right)^{1 / 2} \sigma^{6}+\left(\frac{d}{m}\right)^{1 / 2} \sigma^{-1}\right\} \\
& \leqq A_{6} M(m)\left\{\sigma^{6} m^{1 / 2} \sigma_{-1 / 2}(m)+(4 \pi)^{1 / 2} \sigma^{2} \sigma_{0}(m)\right\}
\end{aligned}
$$

Also, by (5.7), (4.17) and (4.14),

Now, by (4.13),

$$
\begin{aligned}
S_{m}^{\prime \prime} & =\sum_{q \geqq Q}\left|J_{\nu}\left(\frac{\nu Q}{q}\right)\right| \leqq A_{5} \sum_{q \geqq Q} f(Q / q) \\
& \leqq A_{5}\left\{\int_{O}^{\infty} f(Q / u) d u+f(1)\right\} \\
& =A_{5}\left\{Q \int_{0}^{1} x^{-2} f(x) d x+\sigma^{2}\right\} \\
& \leqq A_{5}\left\{Q \int_{0}^{1} x^{-2} F(x) d x+\sigma^{2}\right\} .
\end{aligned}
$$

$$
\int_{0}^{1} x^{-2} F(x) d x=A_{3} I_{1}+A_{4} I_{2}
$$

where

$$
I_{1}=\int_{0}^{x_{0}} \sigma^{3} x^{-2}\left(\frac{1}{2} e x\right)^{\nu} d x
$$

and

$$
I_{2}=\int_{x_{0}}^{1} \sigma^{3} x^{-2}\left(1-x^{2}\right)^{-1 / 4} \exp \left\{-\frac{1}{3} \nu\left(1-x^{2}\right)^{3 / 2}\right\} d x
$$


Hence

$$
I_{1}=\frac{\sigma^{3}\left(\frac{1}{2} e x_{0}\right)^{\nu}}{(\nu-1) x_{0}}
$$

note that $\frac{1}{2} e x_{0}<0 \cdot 9$. Also

$$
\begin{aligned}
I_{2} & \leqq\left(\frac{\sigma}{x_{0}}\right)^{3} \int_{x_{0}}^{1} x\left(1-x^{2}\right)^{-1 / 4} \exp \left\{-\frac{1}{3} \nu\left(1-x^{2}\right)^{3 / 2}\right\} d x \\
& \leqq \frac{\sigma^{6}}{x_{0}^{3} \sqrt{3}} \int_{1}^{\infty} t^{-1 / 2} e^{-t} d t=\left(\frac{\pi}{3}\right)^{1 / 2} \frac{\sigma^{6}}{x_{0}^{3}}
\end{aligned}
$$

Accordingly, by (5.7) and (5.12),

if $m \leqq \nu^{2}$.

$$
S_{m}^{\prime \prime} \leqq A_{5} \sigma^{2}+A_{7} m \sigma^{15}\left(\frac{1}{2} e x_{0}\right)^{\nu}+A_{8} m \sigma^{12} \leqq A_{9} m \sigma^{12},
$$

From (5.5), (5.11), (5.13) and (3.9) we deduce that

$$
\left|S_{m}\right| \leqq A_{6} m^{1 / 2} \sigma^{6} M(m) \sigma_{-\frac{1}{2}}(m)+A_{10} \sigma^{2} M^{2}(m)+A_{9} m \sigma^{12}
$$

provided that $m \leqq \nu^{2}$. We now restrict $m$ further by taking it to satisfy (1.6) (recall that $k=\nu+1)$, where $B>4 B_{1}$. The second and third terms on the right of (5.14) are then clearly $o(1)$ for large $k$, while the first term is, by (3.10),

$$
A_{6} \frac{k}{\nu} \exp \left\{\frac{\left(4 B_{1}-B\right) \log k}{2 \log \log k}(1+o(1))\right\} \text {. }
$$

It follows that

$$
\left|S_{m}\right|<\frac{1}{2 \pi}
$$

for sufficiently large $k$. Hence, by $(2.7), c_{k}(m, m)>0$ and this completes the proof of Theorem 1.

\section{Further results}

We require the following lemma:

Lemma 6.1. (i) If $c_{k}(m, m)=0$, then $c_{k}(m n, m n)=0$ whenever $(m, n)=1$.

(ii) If $c_{k}(m, m) \neq 0$ and $p$ is any prime not dividing $m$, then, for each integer $\mu \geqq 1$, either (a) $c_{k}\left(m p^{\mu}, m p^{\mu}\right) \neq 0$ or (b) $c_{k}\left(m p^{\mu-1}, m p^{\mu+1}\right) \neq 0$.

Proof. By repeated applications of (3.2) we find, when $p+m$ and $\mu \geqq 1$,

$$
c_{k}\left(m p^{\mu}, m p^{\mu}\right)=\sum_{\lambda=0}^{\mu} p^{(k-1) \lambda} c_{k}\left(m, m p^{2(\mu-\lambda)}\right) .
$$

Similarly, when $\mu \geqq 2$,

$$
c_{k}\left(m p^{\mu-1}, m p^{\mu+1}\right)=\sum_{\lambda=0}^{\mu-1} p^{(k-1) \lambda} c_{k}\left(m, m p^{2(\mu-\lambda)}\right)
$$


Note that this is also trivially true when $\mu=1$. From these two formulae we deduce that, when $\mu \geqq 1$,

$$
c_{k}\left(m p^{\mu}, m p^{\mu}\right)=p^{(k-1) \mu} c_{k}(m, m)+c_{k}\left(m p^{\mu-1}, m p^{\mu+1}\right) .
$$

Thus, if $c_{k}(m, m)=0$ and also $c_{k}\left(m p^{\mu-1}, m p^{\mu-1}\right)=0$ for any $\mu \geqq 1$, then $g_{k}\left(z, m p^{\mu-1}\right) \risingdotseq 0$ and so the right-hand side of $(6.1)$ vanishes. It follows that $c_{k}\left(m p^{\mu}, m p^{\mu}\right)=0$. By induction on $\mu(\mu=1,2,3, \ldots)$, we deduce that

$$
c_{k}(m, m)=0 \Rightarrow c_{k}\left(m p^{\mu}, m p^{\mu}\right)=0 \text { for all } \mu \geqq 0,
$$

and part (i) of the lemma follows from this.

Moreover, if $c_{k}(m, m) \neq 0$, it follows from (6.1) that it is not possible for both $c_{k}\left(m p^{\mu}, m p^{\mu}\right)$ and $c_{k}\left(m p^{\mu-1}, m p^{\mu+1}\right)$ to be zero. This gives part (ii).

From Lemma 6.1 and (2.5) we deduce

Theorem 2. (i) If $g_{k}(z, m) \equiv 0$, then, for any positive integer $n$ prime to $m$,

$$
g_{k}(z, m n) \equiv 0 .
$$

(ii) If $g_{k}(z, m) \neq 0$ and if $p$ is a prime not dividing $m$ and $\mu \geqq 1$, then (a) $g_{k}\left(z, m p^{\mu}\right) \not \equiv 0$, or (b) $g_{k}\left(z, m p^{\mu-1}\right) \not \equiv 0$ and $g_{k}\left(z, m p^{\mu+1}\right) \not \equiv 0$.

As a corollary of part (ii) of the theorem we note that it follows that, if $g_{k}(z, m) \neq 0$, then it is not possible for $g_{k}\left(z, m p^{\mu}\right)$ to be identically zero for two consecutive positive integers $\mu$.

Theorem 2(i) can also be deduced from the relation

$$
g_{k}(z, m)\left|T_{n}=g_{k}(z, n)\right| T_{m}=\sum_{d \mid(m, n)} d^{k-1} g_{k}\left(z, m n / d^{2}\right) ;
$$

see equation (23) of (3), or Theorem 9.3.1 of (5). Here $T_{n}$ and $T_{m}$ are Hecke operators.

From (6.2) we also deduce

Theorem 3. For any positive integer $m$,

$$
g_{k}(z, m) \equiv 0 \text { if and only if } T_{m} \text { annihilates } C_{k} .
$$

Proof. That $g_{k}(z, m) \equiv 0$ implies that $C_{k} \mid T_{m}=0$ follows immediately from the first equation in (6.2). Conversely, if $C_{k} \mid T_{m}=0$ then

$$
g_{k}(z, m)=g_{k}(z, 1) \mid T_{m}=0 .
$$

In conclusion, we remark that the formula (3.1) is related to the formula

$$
S(m, n ; q)=\sum_{d \mid(m, n, q)} d S\left(1, \frac{m n}{d^{2}} ; \frac{q}{d}\right)
$$

quoted by Selberg (6). He mentions that the multiplicative properties of cusp form coefficients such as $\tau(n)$ can easily be deduced from (6.3), but gives no details. In this connexion it may be of interest to record that the formula (3.2) was used by the author in his $\mathrm{Ph} . \mathrm{D}$. thesis (1940) to prove multiplicative properties of this kind. 


\section{Postscript}

It is possible that recent work of N. V. Kuznetsov on the order of magnitude of partial sums of the form

$$
\sum_{0<c \leqq T} \frac{1}{c} S(m, n ; c)
$$

may enable Theorem 1 to be improved. This is referred to in the preprint entitled "Peterson's hypothesis for forms of zero weight and Linnik's hypothesis" (Akad. Nauk SSSR, Khabarovsk, 1977).

\section{REFERENCES}

(1) T. EstermanN, On Kloosterman's sum, Mathematika 8 (1961), 83-86.

(2) R. E. LANGER, On the asymptotic solutions of ordinary differential equations, with an application to the Bessel functions of large order, Trans. Amer. Math. Soc. 33 (1931), 23-64.

(3) H. Petersson, Über eine Metrisierung der ganzen Modulformen, Jber. Deutsch. Math.-Verein. 49 (1939), 49-75.

(4) R. A. Rankin, The scalar product of modular forms, Proc. London Math. Soc. (3) 2 (1952), 198-217.

(5) R. A. RANKIN, Modular forms and functions (Cambridge, 1977).

(6) A. SELBERG, Über die Fourierkoeffizienten elliptischer Modulformen negativer Dimension, Neuvième Congrès des Mathematiciens Scandinaves, Helsingfors, 1938, pp. 320-2.

(7) G. N. Watson, A treatise on the theory of Bessel functions (Cambridge, 1922).

DEPARTMENT OF MATHEMATICS

UNIVERSITY OF GLASGOW 